\title{
Síntesis y evaluación "in vitro" de la actividad antifúngica de oximas, éteres de oxima e isoxazoles
}

\author{
John Diaz-Velandia, Natalia Durán-Díaz, Jorge Robles-Camargo, Alix Elena Loaiza* \\ Laboratorio de síntesis orgánica, Departamento de Química. Facultad de Ciencias, Pontificia Universidad Javeriana, Bogotá, D.C., Colombia. \\ *aloaiza@javeriana.edu.co
}

Recibido: 23-09-2011; Aceptado: 08-11-2011

\begin{abstract}
Resumen
Objetivo. Sintetizar y realizar la evaluación preliminar de la actividad antifúngica in vitro de oximas, éteres de oxima e isoxazoles. Materiales y métodos. Las oximas se sintetizaron a partir de aldehídos o cetonas con $\mathrm{NH}_{2} \mathrm{OH} . \mathrm{HCl}_{\text {y }} \mathrm{K}_{2} \mathrm{CO}_{3}$. Los éteres de oxima se obtuvieron mediante alquilación de oximas con bromuro de propargilo o bromuro de 2-bromobencilo, empleando como base $\mathrm{NaOH}$ y acetona como solvente. Los isoxazoles se obtuvieron mediante cicloadiciones 1,3-dipolares empleando nitrato cérico amónico (NAC), cloramina-T (CAT) y NaOCl. Los productos fueron identificados y/o caracterizados por resonancia magnética nuclear (RMN) y espectrometría de masas (EM). Se realizaron pruebas de inhibición de crecimiento radial sobre Aspergillus niger y Fusarium roseum. Resultados. Se obtuvieron cinco oximas, siete éteres de oxima, cuatro de ellos nuevos y cuatro nuevos isoxazoles. Las sustancias evaluadas presentaron actividad antifúngica a cantidades de 1,5 mg y 3,0 mg. Conclusiones. Aunque las cicloadiciones 1,3-dipolares permitieron obtener los isoxazoles esperados, se observó que ésta metodología generó una amplia variedad de subproductos lo que disminuyó los rendimientos e hizo difícil la purificación del producto de interés. Cuatro de las sustancias evaluadas presentaron porcentajes de inhibición superiores al $80 \%$.
\end{abstract}

Palabras clave: oximas, éteres de oxima, isoxazoles, actividad antifúngica.

\begin{abstract}
Synthesis and in vitro assessment of antifungal activity of oximes, oxime ethers and isoxazoles. Objective. To synthesize and carry out a preliminary evaluation of the in vitro antifungal activity of oximes, oxime ethers and isoxazoles. Materials and methods. Oximes were synthesized from aldehydes or ketones with $\mathrm{NH}_{2} \mathrm{OH} . \mathrm{HCl}$ and $\mathrm{K}_{2} \mathrm{CO}_{3}$. Oxime ethers were prepared by alkylation of oximes with propargyl bromide or 2-bromobenzyl bromide, using $\mathrm{NaOH}$ as base and acetone as solvent. The isoxazoles were obtained by 1,3-dipolar cycloadditions using ceric ammonium nitrate (CAN), chloramine T (CAT) and NaOCl. Products were identified or characterized using nuclear magnetic resonance (NMR) and mass spectrometry (MS). Radial growth inhibition assays against Aspergillus niger and Fusarium roseum were carried out. Results. Five oximes, seven oxime ethers, four of them new, and four new isoxazoles were obtained. The assessed substances exhibited antifungal activity in amounts of 1,5 mg and 3,0 mg. Conclusions. Although 1,3-dipolar cycloadditions allowed to obtain the desired isoxazoles, this methodology produced a wide variety of side products that reduced yields and made difficult the purification of the target products. Four of the tested compounds showed inhibition percentages greater than $80 \%$.
\end{abstract}

Key words: oximes, oxime ethers, isoxazoles, antifungal activity.

\section{Resumo}

Síntese e avaliação "in vitro" da atividade antifúngica de oximas, éteres de oxima e isoxazóis. Objetivo. Sintetizar e realizar a avaliação preliminar da atividade antifúngica in vitro de oximas, éteres de oxima e isoxazóis. Materiais e métodos. As oximas foram sintetizadas a partir de aldeídos ou cetonas com $\mathrm{NH}_{2} \mathrm{OH} . \mathrm{HCl}$ e $\mathrm{K}_{2} \mathrm{CO}_{3}$. Os éteres de oxima foram obtidos pela alquilação de oximas com brometo de propargilo ou brometo de 2-bromobenzilo, utilizando $\mathrm{NaOH}$ como base e acetona como solvente. Os isoxazóis foram obtidos 
por cicloadição 1,3-dipolar usando nitrato cérico de amônio (NCA), cloramina-T (CAT) e NaOCl. Os produtos foram identificados e / ou caracterizados por ressonância magnética nuclear (RMN) e espectrometria de massas (EM). Foram realizados testes de inibição sobre o crescimento radial de Aspergillus niger e Fusarium roseum. Resultados. Foram obtidas cinco oximas, sete éteres de oxima, quatro deles novos e quatro novos isoxazóis. As substâncias testadas apresentaram atividade antifúngica em quantidades de 1,5 mg e 3,0 mg. Conclusões. Embora as cicloadições 1,3-dipolares permitiram obter os isoxazóis esperados, observou-se que esta metodologia resultou numa grande variedade de subprodutos que reduziram os rendimentos e tornaram difícil a purificação do produto de interesse. Quatro das substâncias testadas apresentaram porcentagens de inibição acima de $80 \%$.

Palavras-chave: oximas, éteres de oxima, isoxazóis, atividade antifúngica.

\section{Introducción}

Las oximas, éteres de oxima e isozaxoles, son compuestos orgánicos que dentro de su estructura poseen átomos de nitrógeno y oxígeno de forma consecutiva. Este tipo de sustancias tienen amplias aplicaciones a nivel químico y biológico, algunas de las cuales se describen a continuación. La función oxima es un intermediario sintético importante ya que puede ser transformada en grupos orgánicos como carbonilo, amino, nitro y ciano; además, es utilizada como grupo protector y en la producción de compuestos heterocíclicos, aprovechando su conversión a óxidos de nitrilo o nitrosoalquenos, intermediarios reactivos, que en presencia de alquenos o alquinos generan isoxazoles y 1,2-oxazinas (1, 2). Muchos compuestos que contienen un fragmento oxima o éter de oxima exhiben diversas actividades biológicas entre las que se encuentran antiinflamatoria (3), antifúngica, antibacterial, anti-cancerígena y antiviral entre otras $(4,5)$.

Los isoxazoles por su parte son compuestos cíclicos de cinco miembros con nitrógeno y oxígeno, miscibles en agua y con olor similar a los compuestos piridínicos, generalmente son sólidos con altos puntos de fusión debido a su fuerte asociación dipolar, de ellos existen heterociclos 4,5-dihidro y tetrahidro, también llamados isoxazolinas e isoxazolidinas. Son importantes intermediarios sintéticos ya que se pueden considerar como aminoalcoholes enmascarados, los cuales hacen parte de una amplia variedad de compuestos biológicamente activos, tales como el ácido iboténico, el muscimol y la acivicina; asimismo tras algunas transformaciones simples pueden dar origen a iminas cíclicas, lactamas, $\beta$-hidroxicetonas, nitrilos y cetonas $\alpha, \beta$-insaturadas (6).

La importancia biológica de los isoxazoles radica en la amplia variedad de actividades que presentan entre las que se reportan analgésica (7), anti-fúngica (8), antiviral (9), antiinflamatoria (10), entre otras.

\section{Materiales y métodos}

En la síntesis de los compuestos, el seguimiento de las reacciones, fue realizado por cromatografía de capa delgada (CCD), empleando soluciones de ácido fosfomolíbdico, vainillina, Dragendorff, luz UV y vapores de yodo como reveladores para su visualización. El aislamiento y purificación se hizo por cromatografía de columna (CC) empacada con sílica gel 60-200 y 230-400 mesh y cromatografía de capa delgada preparativa (CCDP). Los productos fueron identificados por la técnica de CG-EM en un cromatógrafo Agilent 6850 serie II, acoplado a un espectrómetro de masas Agilent 5975B VL MSD equipado con un puerto de inyección split/splitess $\left(260^{\circ} \mathrm{C}\right.$, relación de split 15:1), con inyector automático Agilent 6850 series y una columna Agilent 19091S-433E HP-5MS. El método utilizado consta de una temperatura inicial del horno de $80^{\circ} \mathrm{C}$ por 1 minuto y luego una rampa de temperatura de $10^{\circ} \mathrm{C} / \mathrm{min}$ hasta $320^{\circ} \mathrm{C}$, la temperatura se mantuvo 3 minutos, para un tiempo de corrida de 28 minutos. La caracterización se realizó por técnicas espectroscópicas convencionales como RMN ${ }^{1} \mathrm{H} \mathrm{y}{ }^{13} \mathrm{C}$, utilizando espectrómetros Brucker de $400 \mathrm{MHz}$ y $300 \mathrm{MHz}$. Los desplazamientos químicos ( $\delta$ ) de protones y carbonos se reportan en ppm relativo a $\mathrm{CDCl}_{3}(7.27$ y 77.26) y las constantes de acoplamiento $(J)$ en Hertz (Hz). Los puntos de fusión se determinaron con un fusiómetro MEL-TEMP Laboratory Devices Mass 02139. Las temperaturas se expresan en ${ }^{\circ} \mathrm{C}$.

\section{Procedimiento utilizado para la síntesis de oximas}

En un balón de fondo redondo a temperatura ambiente se agitó durante diez minutos una mezcla de $1 \mathrm{mmol}$ de aldehído o cetona, 1,3 mmol de clorhidrato de hidroxilamina en 1,1 $\mathrm{mL}$ de agua. Se agregaron $0,5 \mathrm{mmol}$ de $\mathrm{K}_{2} \mathrm{CO}_{3}$ en solución acuosa 0.3 molar, y se dejó por 30 minutos en agitación. El producto sólido se filtró en un embudo Gooch y se secó a temperatura ambiente $(1,3-5)$.

\section{Procedimiento utilizado para la síntesis de éteres de oxima}

En un balón de dos bocas se mezclaron $1 \mathrm{mmol}$ de oxima, $2 \mathrm{mmol} \mathrm{NaOH}$ en $1,43 \mathrm{~mL}$ de acetona a reflujo durante 30 minutos, luego se le agregó $1,1 \mathrm{mmol}$ de bromuro de propargilo ó bromuro de 2-bromobencilo. La mezcla se dejó reaccionar durante 3 horas, luego de las cuales se neutralizó con ácido acético, se extrajo en acetato de etilo, se secó con $\mathrm{Na}_{2} \mathrm{SO}_{4}$, se concentró a presión reducida y se purificó $(1,3-5)$. 


\section{Procedimiento utilizado para la síntesis de isoxazoles con $\mathrm{NaOCl}$}

Se mezclaron $1 \mathrm{mmol}$ de aldoxima y $2 \mathrm{mmol}$ de dipolarófilo (alqueno o alquino) en $4 \mathrm{~mL}$ de $\mathrm{CH}_{2} \mathrm{Cl}_{2}$. La mezcla se colocó en un baño de hielo y se agitó durante 5 minutos. Se agregaron $3 \mathrm{~mL}(2,5 \mathrm{mmol})$ de $\mathrm{NaOCl}$ al $5,25 \%$ en solución acuosa, hasta desaparición del material de partida. Se separaron las fases y se extrajo la fase acuosa con AcOEt, se secó con $\mathrm{Na}_{2} \mathrm{SO}_{4}$, se concentró a presión reducida y se purificó mediante $\mathrm{CC}$.

\section{Procedimiento utilizado para la síntesis de isoxazoles con NAC}

Se mezcló $1 \mathrm{mmol}$ de aldoxima y $2 \mathrm{mmol}$ de dipolarófilo en $5,4 \mathrm{~mL}$ de acetonitrilo y se agitó hasta obtener una mezcla homogénea. Luego se agregó 2,2 mmol de NAC, se dejó en agitación hasta la aparición de nuevos productos por $\mathrm{CCD}$, se filtró a través de sílica con el fin de eliminar los óxidos de cerio, se secó con $\mathrm{Na}_{2} \mathrm{SO}_{4}$, se concentró a presión reducida y se purificó.

\section{Procedimiento utilizado para la síntesis de isoxazoles con CAT}

Se mezcló $1 \mathrm{mmol}$ de aldoxima y $2 \mathrm{mmol}$ de dipolarófilo en 7,9 mL de etanol, y se agitó hasta obtener una mezcla homogénea. Luego se agregó $1 \mathrm{mmol}$ de CAT, se llevó la mezcla a temperatura de reflujo con agitación constante hasta desaparición del material de partida según CCD, se concentró a presión reducida y se purificó. $(6,8$-10)

\section{Determinación de la actividad antifúngica sobre hongos fitopatógenos}

La actividad antifúngica preliminar se determinó mediante pruebas de inhibición de crecimiento radial (11).

\section{Obtención de las cepas}

Las cepas Fusarium roseum y Aspergillus niger, fueron obtenidas del cepario de hongos de la Facultad de Ciencias en la Pontifica Universidad Javeriana sede Bogotá. En su recuperación se utilizó medio PDA y se confirmó la viabilidad y pureza a partir de evaluaciones tanto macroscópica como microscópica (12).

\section{Pruebas preliminares de porcentaje de inhibición de crecimiento radial}

Se recuperó cada uno de los hongos fitopatógenos a evaluar en agar PDA, los cuales después de 5 días de crecido para Aspergillus niger y 7 días para Fusarium roseum se tomaron discos de $5 \mathrm{~mm}$ de diámetro y se inocularon en Agar Mueller-
Hinton modificado (11), dónde previamente se agregaron cantidades de $1,5 \mathrm{mg}$ y $3,0 \mathrm{mg}$ de cada compuesto y se dejó secar en nevera por 2 días, luego se incubaron por 96 horas a una temperatura de $28^{\circ} \mathrm{C}$. Los ensayos se realizaron por triplicado. Se realizó lectura de los mm de crecimiento radial a las 24, 48, 72 y 96 horas, porque los hongos en el control negativo habían crecido más del $80 \%$, lo que permitía detectar la actividad (13).

Se halló el área bajo la curva para determinar el porcentaje de inhibición de crecimiento radial de cada réplica para todas las lecturas realizadas, se promediaron los porcentajes y se realizó estadística descriptiva, ANOVA de una vía y prueba de Duncan. Se utilizó como control positivo la terbinafina por su conocida acción antifúngica, especialmente sobre hongos filamentosos del género Fusarium (14), a una concentración de $2.5 \mathrm{mg} / \mathrm{mL}$ e igualmente se realizaron controles negativos para cada una de las cepas.

\section{Resultados y discusión}

\section{Síntesis de oximas 1 y éteres de oxima 2 y 3}

La síntesis de las oximas 1a-e (etapa i, Figura 1) se realizó mediante la condensación de los grupos carbonilos del nicotinaldehído, benzaldehído, cinamaldehído, ciclohexanona y 1-bencil-4-piperidona con clorhidrato de hidroxilamina en medio básico. Los éteres de oxima $\mathbf{2}$ y $\mathbf{3}$ se obtuvieron por alquilación del oxigeno del grupo $\mathrm{OH}$ de la oxima con bromuro de propargilo (ii) y bromuro de 2-bromobencilo (iii) respectivamente, empleando como base $\mathrm{NaOH}$ (15).

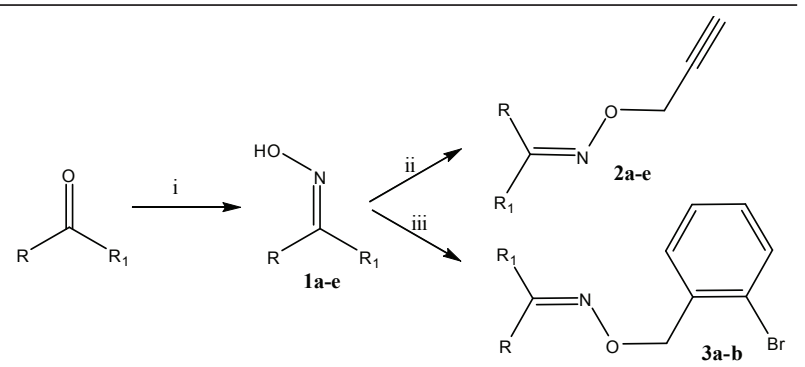

a: $\mathrm{R}=\mathrm{R}_{1}=\mathrm{CH}_{2}\left(\mathrm{CH}_{2}\right)_{3} \mathrm{CH}_{2} ; \mathbf{b}: \mathrm{R}=3-\mathrm{py}, \mathrm{R}_{1}=\mathrm{H} ; \mathbf{c}: \mathrm{R}=\mathrm{C}_{6} \mathrm{H}_{5}$, $\mathrm{R}_{1}=\mathrm{H} ; \mathbf{d}: \mathrm{R}=\mathrm{Cinm}, \mathrm{R}_{1}=\mathrm{H} ; \mathbf{e}: \mathrm{R}=\mathrm{R}_{1}=\left(\mathrm{CH}_{2}\right)_{2} \mathrm{NBn}\left(\mathrm{CH}_{2}\right)_{2}$

Figura 1. Ruta empleada para la síntesis de oximas y éteres de oxima. Condiciones: i) $\mathrm{HONH}_{2} \cdot \mathrm{HCl} / \mathrm{H}_{2} \mathrm{O}$, t.a. ii) $\mathrm{NaOH} /$ Bromuro de propargilo, $60{ }^{\circ} \mathrm{C}$ iii) $\mathrm{NaOH} /$ Bromuro de 2-bromobencilo, $60{ }^{\circ} \mathrm{C}$

Las oximas 1a-e se obtuvieron con rendimientos entre 65$96 \%$. Los puntos de fusión determinados experimentalmente para éstas oximas coinciden con lo reportado en la literatura para cada sustancia (Tabla 1). 
Tabla 1. Rendimiento y puntos de fusión de las oximas 1a-e

\begin{tabular}{lccc}
\hline \multicolumn{1}{c}{ Compuesto } & Número & Rendimiento & Punto de fusión \\
\hline Oxima de la ciclohexanona & $\mathbf{1 a}$ & $75 \%$ & $89-90^{\circ} \mathrm{C}$ \\
Nicotinaldoxima & $\mathbf{1 b}$ & $86 \%$ & $151-152^{\circ} \mathrm{C}$ \\
Benzaldoxima & $\mathbf{1 c}$ & $81 \%$ & $31{ }^{\circ} \mathrm{C}$ \\
Oxima del cinamaldehído & $\mathbf{1 d}$ & $65 \%$ & $110-115^{\circ} \mathrm{C}$ \\
Oxima de la 1-bencil-4-piperidona & $\mathbf{1 e}$ & $96 \%$ & $129-131^{\circ} \mathrm{C}$ \\
\hline
\end{tabular}

Tabla 2. Desplazamientos químicos, multiplicidad y constantes de acoplamientos de algunos protones de los éteres de oxima 2a-e y 3a-b en $\mathrm{RMN}-{ }^{1} \mathrm{H}\left(\mathrm{CDCl}_{3}, 300 \mathrm{MHz}\right)$.

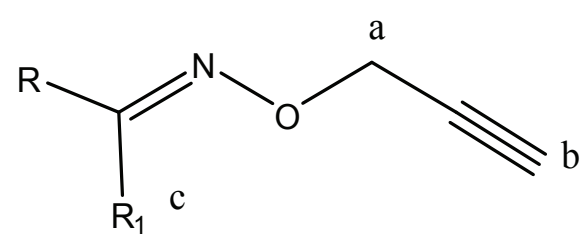

2a. $\mathrm{R}=\mathrm{R}_{1}=\mathrm{CH}_{2}\left(\mathrm{CH}_{2}\right)_{3} \mathrm{CH}_{2}$

2b.R $=3$-py, $\mathrm{R}_{1}=\mathrm{H}$

2c. $\mathrm{R}=\mathrm{C}_{6} \mathrm{H}_{5}, \mathrm{R}_{1}=\mathrm{H}$

2d. $\mathrm{R}=$ Cinm, $\mathrm{R}_{1}=\mathrm{H}$

2e. $\mathrm{R}=\mathrm{R}_{1}=\left(\mathrm{CH}_{2}\right)_{2} \mathrm{NBn}\left(\mathrm{CH}_{2}\right)_{2}$

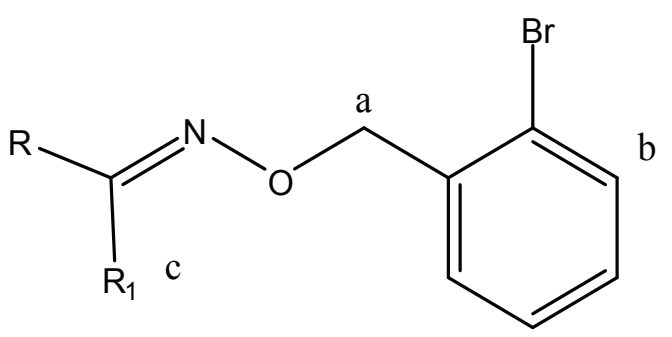

3a. $\mathrm{R}=\mathrm{R}_{1}=\mathrm{CH}_{2}\left(\mathrm{CH}_{2}\right)_{3} \mathrm{CH}_{2}$

3b.R=3-py, $\mathrm{R}_{1}=\mathrm{H}$

\begin{tabular}{cccc}
\hline Compuesto & Ha & Hb & Hc \\
\hline $\mathbf{2 a}$ & $4,56, \mathrm{~d},(3 \mathrm{~Hz})^{*}$ & $2,41, \mathrm{t},(3 \mathrm{~Hz})$ & $2,44, \mathrm{t},(6 \mathrm{~Hz})^{*}$ \\
\hline $\mathbf{2 b}$ & $4,81, \mathrm{~d},(3 \mathrm{~Hz})^{*}$ & $2,53, \mathrm{t},(3 \mathrm{~Hz})$ & $8,15, \mathrm{~s}$ \\
\hline $\mathbf{2 c}$ & $4,80, \mathrm{~d},(2,4 \mathrm{~Hz})^{*}$ & $2,53, \mathrm{t},(2,4 \mathrm{~Hz})$ & $8,15, \mathrm{~s}$ \\
\hline $\mathbf{2 d}$ & $4,77, \mathrm{~d},(2,4 \mathrm{~Hz})^{*}$ & $2,53, \mathrm{t},(2,4 \mathrm{~Hz})$ & $7,81, \mathrm{~d},(12 \mathrm{~Hz})$ \\
\hline $\mathbf{2 e}$ & $4,62, \mathrm{~d},(3 \mathrm{~Hz})^{*}$ & $2,46, \mathrm{t},(3 \mathrm{~Hz})$ & $2,51, \mathrm{~m} *$ \\
\hline $\mathbf{3 a}$ & $5,16, \mathrm{~s}^{*}$ & $7,59, \mathrm{dd},(7 \mathrm{~Hz})$ & $2,57, \mathrm{t},(6 \mathrm{~Hz})^{*}$ \\
\hline $\mathbf{3 b}$ & $5,33, \mathrm{~s}^{*}$ & $7,59, \mathrm{dd},(5 \mathrm{~Hz} ; 0,8 \mathrm{~Hz})$ & $8,19, \mathrm{~s}$ \\
\hline
\end{tabular}

*señales que integran para dos protones equivalentes. 
Los éteres de oxima 2a-e y 3a,b, se obtuvieron con rendimientos entre el $50-83 \%$, sus estructuras fueron confirmadas por RMN y EM. Cuatro de los éteres de oxima sintetizados son sustancias que no han sido reportadas con anterioridad, éstas son la O-2-propiniloxima del cinamaldehído (2d) y la O-2-propinil oxima de la 1-bencil4-piperidona (2e), además de los productos O-2-bromobencil oxima del nicotinaldehído (3b) y O-2-bromobencil oxima de la ciclohexanona (3a).

El compuesto 3b tiene la fórmula molecular $\mathrm{C}_{13} \mathrm{H}_{11} \mathrm{~N}_{2} \mathrm{OBr}$, con un peso molecular 291,14. Su espectro de masas, presenta dos picos de ión molecular uno en $\mathrm{m} / \mathrm{z} 290,0$ y el otro en $\mathrm{m} / \mathrm{z}$ 292,0 de intensidad similar debido a que el bromo presenta dos isótopos mayoritarios ${ }^{79} \mathrm{Br} \mathrm{y}^{81} \mathrm{Br}$ en proporciones muy parecidas, por la misma causa presenta dos picos base en $\mathrm{m} / \mathrm{z}, 169,0$ y $\mathrm{m} / 2.171,0$ debidos al catión bromobencilo, entre otras fragmentaciones usuales de compuestos aromáticos. En el espectro $\mathrm{RMN}-{ }^{1} \mathrm{H}$ de $\mathbf{3 b}$ las integrales muestran la existencia de once protones, de los cuales, nueve presentan desplazamientos entre 7,0 y 8,8 ppm (región aromática); según sus multiplicidades y constantes de acoplamiento corresponden a dos anillos diferentes, lo cual fue confirmado con el experimento COSY $-{ }^{1} \mathrm{H},{ }^{1} \mathrm{H}$.

El espectro APT de RMN $-{ }^{13} \mathrm{Cmuestra}$ que la sustancia presenta trece carbonos, entre los que se encuentran tres cuaternarios, nueve grupos $\mathrm{CH}$ y un $\mathrm{CH}_{2}$. Las señales más desplazadas a campo bajo corresponden al $\mathrm{C}=\mathrm{N}$ del grupo oxima y a los carbonos alfa al nitrógeno en el anillo piridínico, mientras que, los carbonos con menor desplazamiento son el carbono cuaternario enlazado al bromo y el $\mathrm{CH}_{2}$ alfa al oxígeno.
Los espectros RMN- $-1 \mathrm{H}$ y $\mathrm{RMN}-{ }^{13} \mathrm{C}$ de los éteres de oxima 2a-e y 3a-b muestran señales a desplazamientos químicos muy similares, por ejemplo en $\mathrm{RMN}^{-1} \mathrm{H}$ los protones del grupo $\mathrm{O}-\mathrm{CH}_{2}-\mathrm{R}$ aparecen entre 4,56 y 5,33 ppm. Por otra parte, el hidrógeno del carbono imínico de los éteres de aldoxima aparece cercano a 8 ppm (Tabla 2).

\section{Síntesis de Isoxazoles 4a-d}

Los isoxazoles 4a-d se sintetizaron mediante reacciones de cicloadición 1,3-dipolares (Figura 2). Para la obtención del isoxazol 4a se probaron tres métodos empleando los reactivos NAC, CAT y $\mathrm{NaOCl}$ siguiendo las condiciones reportadas en la literatura (15-18), y con base en los resultados, se seleccionó el $\mathrm{NaOCl}$ para obtener los isoxazoles $\mathbf{4 b - d}$.

Para la obtención de los óxidos de nitrilo (intermediarios en la formación de isoxazoles), se evaluaron tres condiciones de reacción: la oxidación de aldoximas con nitrato amónico cérico (NAC) en acetonitrilo, y la halogenación-deshidrohalogenación con cloramina-T (CAT) en etanol, e hipoclorito de sodio comercial $(\mathrm{NaOCl} 5,25 \%)$ en diclorometano. Se usaron como materiales de partida la nicotinaldoxima y el ciclohexeno, con el fin de obtener el producto 4a. Estas reacciones fueron monitoreadas por cromatografía de capa delgada y por CG-EM, con el fin de establecer los subproductos de reacción y el rendimiento relativo del isoxazol de interés. En la Tabla 3 se resumen los resultados para las tres condiciones de reacción evaluadas.

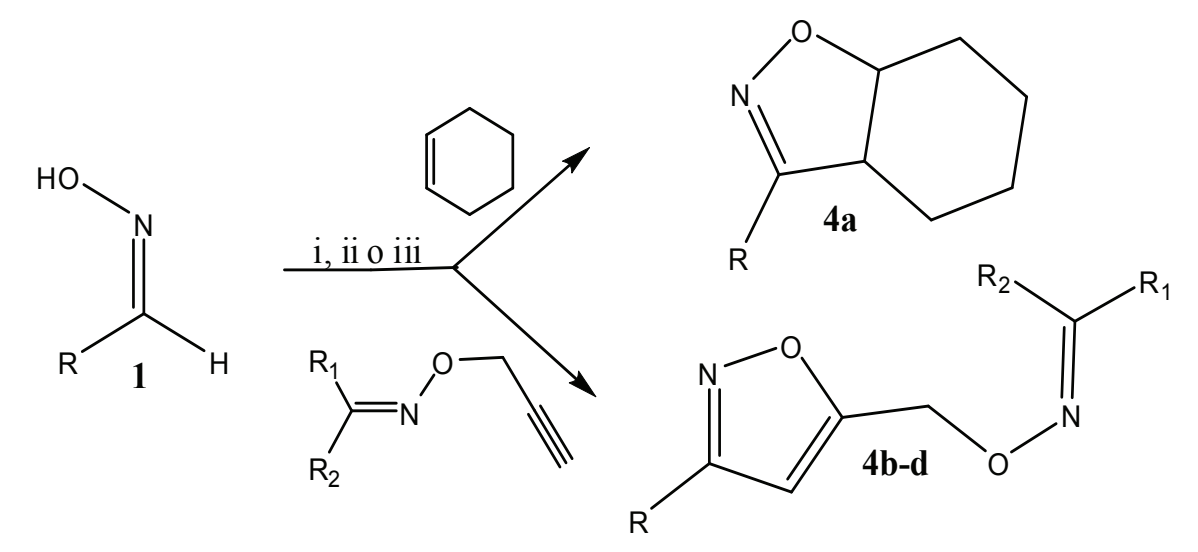

a: $\mathrm{R}=3$-py; b: $\mathrm{R}=\mathrm{C}_{6} \mathrm{H}_{5}, \mathrm{R}_{1}=\mathrm{C}_{6} \mathrm{H}_{5}, \mathrm{R}_{2}=\mathrm{H} ; \mathbf{c}: \mathrm{R}=3-p y, \mathrm{R}_{1}=\mathrm{C}_{6} \mathrm{H}_{5}, \mathrm{R}_{2}=\mathrm{H}$;

d: $\mathrm{R}=3-\mathrm{py}, \mathrm{R}_{1}=\mathrm{R}_{2}=\left(\mathrm{CH}_{2}\right)_{2} \mathrm{NBn}\left(\mathrm{CH}_{2}\right)_{2}$

Figura 2. Síntesis de isoxazoles mediante reacciones de cicloadición 1,3-dipolares. Condiciones i) $\mathrm{NaOCl} / \mathrm{CH}_{2} \mathrm{Cl}_{2} / 15^{\circ} \mathrm{C}$ ii) $\mathrm{NAC} / \mathrm{MeCN} /$ t.a. iii) $\mathrm{CAT} / \mathrm{EtOH}$ reflujo 
Tabla 3. Resultados de la reacción de cicloadición 1,3-dipolar
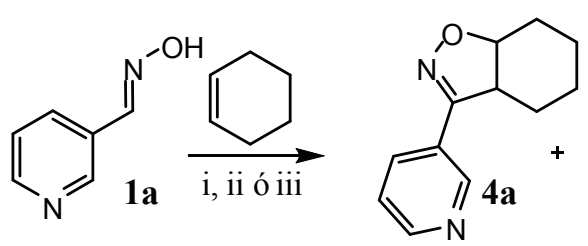

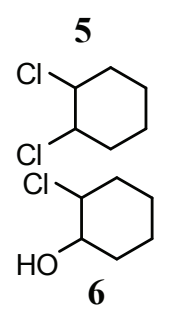<smiles>O=Cc1cccnc1</smiles>

\begin{tabular}{cccc}
\hline Condiciones de Reacción & $\begin{array}{c}\mathbf{N a O C l} / \mathbf{C H}_{\mathbf{2}} \mathbf{C l}_{2} / \mathbf{1 5}{ }^{\circ} \mathbf{C} \\
\text { (i) }\end{array}$ & $\begin{array}{c}\text { NAC/MeCN/t.a. } \\
\text { (ii) }\end{array}$ & $\begin{array}{c}\text { CAT/EtOH/reflujo } \\
\text { (iii) }\end{array}$ \\
\hline $\begin{array}{c}\text { Rendimiento } \\
\text { relativo de 4a }\end{array}$ & $60 \%$ & $30 \%$ & $12 \%$ \\
\hline $\begin{array}{c}\text { Otros productos detectados } \\
\text { por CG-EM }\end{array}$ & $\begin{array}{c}\mathbf{5 , 6} \\
\mathbf{7} \mathbf{9}\end{array}$ & $\mathbf{9}$ y10 & $\begin{array}{c}\mathbf{8 , 9} \mathrm{y} \\
\mathbf{1 0}\end{array}$ \\
\hline $\begin{array}{c}\text { Tiempo de } \\
\text { reacción }\end{array}$ & $1.5-3.0 \mathrm{~h}$ & Días & $1-2 \mathrm{~h}$ \\
\hline
\end{tabular}

Con base en los resultados se seleccionó el $\mathrm{NaOCl} 5,25 \%$ para la síntesis de los isoxazoles $\mathbf{4 b - d}$, debido a la rapidez de las reacciones, el rendimiento del producto de interés y el bajo costo de éste reactivo.

El compuesto 4a se puede considerar como análogo de la nicotina, tiene un peso molecular de 202.1, su espectro de masas presenta como ión molecular y pico base un ion de $m / z$ 202.1, y fragmentaciones en $m / z 185.1$ debido a la pérdida de radical $\mathrm{OH}, \mathrm{m} / \mathrm{z}, 173.1$ pérdida de acetileno, 131.1 debido a la pérdida del radical $\mathrm{C}_{4} \mathrm{H}_{7} \mathrm{O}$, entre otros. En el espectro RMN- ${ }^{1} \mathrm{H}$ se observan los ocho hidrógenos de los grupos metilenos del anillo de ciclohexano entre 1,2 y 2.3 ppm. Los hidrógenos de los grupos $\mathrm{CH}$ en la fusión del anillo isoxazolidínico con el anillo de ciclohexano, muestran la fusión cis esperada en éste tipo de cicloadiciones con desplazamientos de 4.5 y $3.3 \mathrm{ppm}$. En la región aromática se observan 4 hidrógenos correspondientes al anillo piridínico. El espectro APT de RMN $-{ }^{13} \mathrm{C}$ demuestra la existencia de dos carbonos cuaternarios, cuatro $\mathrm{CH}_{2}$ y seis $\mathrm{CH}$.

Las reacciones de cicloadición para la síntesis de los isoxazoles $\mathbf{4 b} \mathbf{b}-\mathbf{d}$, procedieron con buena regioselectividad, pues al realizar el análisis de los productos por RMN y CGEM, sólo se observó la formación del isoxazol sustituido en las posiciones 3 y 5 , razón por la cual sus hidrógenos y carbonos presentan desplazamientos químicos similares como se observa en la Tabla 4.

\section{Evaluación de la actividad antifúngica}

La actividad antifúngica in vitro de 11 de las sustancias sintetizadas fue evaluada, entre estas se encuentran las oximas 1a, c-e, los éteres de oxima $\mathbf{2 a}, \mathbf{b}, \mathbf{d}$ y $\mathbf{3 a}, \mathbf{b}$; la isoxazolina $\mathbf{4 a}$ y el isoxazol con grupo oxima $\mathbf{4 b}$, con los cuales se realizaron pruebas de inhibición de crecimiento radial. Los resultados se pueden observar en la Tabla 5.

Las sustancias que presentaron mayor actividad sobre Aspergillus niger y Fusarium roseum fueron los éteres de oxima O-2-bromobencil nicotinaldoxima (3b) y O-2-propinil oxima del cinamaldehído (2d), adicionalmente contra $A$. niger también inhibieron las oximas del benzaldehído (1c) y cinamaldehído (1d), con porcentajes de inhibición mayores al $90 \%$ comparados con la terbinafina, empleada como control positivo.

Por otra parte, la oxima 3a exhibió actividades de 79\% y $61 \%$ a 3,0 mg contra $F$. roseum y A. niger respectivamente. Ésta oxima al igual que $\mathbf{3 b}$, presenta en su estructura un grupo bromobencilo unido al oxígeno oxímico, el cual, en estudios de relación actividad-estructura $(19,20)$, ha mostrado incrementar la actividad antifúngica de las oximas que lo contienen. Vale la pena anotar que hasta el momento el mecanismo de acción de los éteres de oxima contra hongos es desconocido, contrario a lo que sucede con el modo de acción de agentes antifúngicos de la familia de los azoles (21). 
Tabla 4. Desplazamientos químicos y multiplicidad de algunos protones y carbonos de los Isoxazoles $4 \mathrm{~b}-\mathrm{d}$ en RMN- ${ }^{1} \mathrm{H}$ y RMN- ${ }^{13} \mathrm{C}\left(\mathrm{CDCl}_{3}, 300\right.$ y $\left.75 \mathrm{MHz}\right)$

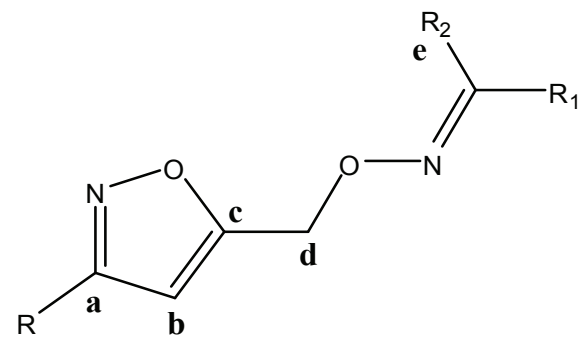

4b: $\mathrm{R}=\mathrm{C}_{6} \mathrm{H}_{5}, \mathrm{R}_{1}=\mathrm{C}_{6} \mathrm{H}_{5}, \mathrm{R}_{2}=\mathrm{H} ; \mathbf{4 c}: \mathrm{R}=3-\mathrm{py}, \mathrm{R}_{1}=\mathrm{C}_{6} \mathrm{H}_{5}, \mathrm{R}_{2}=\mathrm{H} ; \mathbf{4 d}: \mathrm{R}=3-\mathrm{py}, \mathrm{R}_{1}=\mathrm{R}_{2}=\left(\mathrm{CH}_{2}\right)_{2} \mathrm{NBn}_{\left(\mathrm{CH}_{2}\right)_{2}}$

\begin{tabular}{cccccc}
\hline Compuesto & Ha & Hb & Hc & Hd & He \\
\hline $\mathbf{4 b}$ & - & $6,64, \mathrm{~s}$ & - & $5,32, \mathrm{~d}$ & $8,18, \mathrm{~s}$ \\
$\mathbf{4 c}$ & - & $6,69, \mathrm{~s}$ & - & $5,34, \mathrm{~d}$ & $8,19, \mathrm{~s}$ \\
$\mathbf{4 d}$ & - & $6,61, \mathrm{~s}$ & - & $5,18, \mathrm{~d}$ & - \\
& $\mathbf{C a}$ & $\mathbf{C b}$ & $\mathbf{C c}$ & $\mathbf{C d}$ & $\mathbf{C e}$ \\
$\mathbf{4 b}$ & 162,3 & 101,5 & 169,5 & 66,7 & 150,2 \\
$\mathbf{4 c}$ & 159,1 & 101,2 & 170,2 & 66,7 & 150,4 \\
$\mathbf{4 d}$ & 159,8 & 100,7 & 171,0 & 65,6 & 159,0 \\
\hline
\end{tabular}

Tabla 5. Porcentaje de inhibición de crecimiento radial \pm desviación estándar

\begin{tabular}{ccccc}
\hline & \multicolumn{2}{c}{$\boldsymbol{F}$. roseum } & \multicolumn{2}{c}{ A. niger } \\
\hline sustancia & $1,5 \mathrm{mg}$ & $3,0 \mathrm{mg}$ & $1,5 \mathrm{mg}$ & $3,0 \mathrm{mg}$ \\
1a & $36,27 \pm 5,49$ & $36,27 \pm 5,68$ & $2,54 \pm 2,84$ & $16,82 \pm 8,76$ \\
1c & $59,69 \pm 7,39$ & $74,78 \pm 1,48$ & $81,54 \pm 19,47$ & $100,00 \pm 0,00$ \\
1d & $47,65 \pm 6,13$ & $92,63 \pm 1,55$ & $79,93 \pm 7,83$ & $100,00 \pm 0,00$ \\
1e & $20,93 \pm 5,01$ & $40,10 \pm 9,96$ & $10,71 \pm 17,22$ & $14,07 \pm 7,28$ \\
2a & $31,00 \pm 0,53$ & $34,59 \pm 8,88$ & ---- & $26,46 \pm 27,88$ \\
2b & $39,98 \pm 3,61$ & $37,47 \pm 1,65$ & ---- & $46,52 \pm 6,60$ \\
2d & $87,18 \pm 8,00$ & $97,72 \pm 2,85$ & $100,00 \pm 0,00$ & $100,00 \pm 0,00$ \\
3a & $51,90 \pm 3,90$ & $78,98 \pm 6,78$ & $60,99 \pm 1,71$ & $61,10 \pm 5,50$ \\
3b & $79,75 \pm 3,91$ & $83,59 \pm 2,12$ & $100,00 \pm 0,00$ & $100,00 \pm 0,00$ \\
4a & $23,75 \pm 6,15$ & $34,35 \pm 5,58$ & ---- & ---- \\
4b & $43,76 \pm 2,42$ & $30,22 \pm 7,35$ & ---- & $19,96 \pm 14,92$ \\
\hline
\end{tabular}

*Terbinafina $(2.5 \mathrm{mg} / \mathrm{mL})$, Inhibición Crecimiento Radial $=100 \%$ 


\section{Conclusiones}

Aunque en la literatura se reporta la síntesis de isoxazoles mediante cicloadiciones 1,3-dipolares de óxidos de nitrilo con alquenos y alquinos, como un método expedito que permite alcanzar muy buenos rendimiento, en el presente trabajo se encontró que la reacción genera una gran cantidad de subproductos tales como aldehídos, oxadiazoles, furoxanos, ácidos, etc., razón por la cual los rendimientos disminuyen considerablemente y las separaciones cromatográficas se dificultan.

Las oximas, éteres de oxima e isoxazoles evaluados presentan actividad frente a los hongos fitopatogenos $F$. roseum y $A$. niger, en cantidades de $1,5 \mathrm{mg}$ y $3,0 \mathrm{mg}$, siendo la mejor actividad para los compuestos O-2-propinil Oxima del cinamaldehido 2d, O-2-bromobenzil Oxima del nicotinaldehido 3b, Oxima del benzaldehido 1c y Oxima del cinamaldehido $\mathbf{1 d}$.

Todas las sustancias sintetizadas en éste trabajo inhiben el crecimiento normal de los hongos evaluados, no obstante, se observó que las oximas y los éteres de oxima presentan porcentajes de inhibición superiores comparados con el isoxazol 4a, lo cual motiva a continuar la búsqueda de nuevas oximas y éteres de oxima, con actividad antifúngica marcada que eventualmente puedan ser aplicadas en el tratamiento de enfermedades.

\section{Agradecimientos}

Los autores agradecen a la Pontifica Universidad Javeriana por la financiación otorgada para realizar ésta investigación y a los profesores del Departamento de Química por la colaboración, especialmente a C. Sánchez y R. Vera.

\section{Financiación}

Éste proyecto fue financiado por la Pontificia Universidad Javeriana a través del proyecto de investigación 00003408.

\section{Conflicto de intereses}

Los autores manifiestan no tener conflicto de intereses.

\section{Referencias}

1. Mikhaleva A, Zaitsev AB, Trofimov BA. Oximes as reagents. Russian Chemical Reviews 2006; 75 (9): 797-823.

2. Lokanatha KM. Heterocycles via Oxime Cycloadditions. Topics in Heterocyclic chemistry 2008; 13, 1-69
3. Sikharulidze MI, Nadaraia NS, Kakhabrishvili ML, Barbakadze NN, Mulkidzhanyan KG. Synthesis and biological activity of several steroidal oximes. Chemistry of Natural Compounds 2010; 46 (3): 493-494.

4. Abele E, Abele R, Golomba L, Višnevska J, Beresneva T, Rubina K,. Lukevics E. Oximes of six-membered heterocyclic compounds with two and three heteroatoms: II. reactions and biological activity. Chemistry of Heterocyclic Compounds 2010; 46 (8): 1123-1153.

5. Abele E, Abele R, Rubina K, Lukevics E. Quinoline Oximes: Synthesis, reactions, and biological activity. Chemistry of Heterocyclic Compounds 2005; 41 (2):163-190.

6. Giomi D, Cordero FM, Pisaneschi, F, Brandi, A. Isoxazoles in Comprehensive Heterocyclic Chemistry III 2008; 4, 365-486.

7. Meyers MJ, Long SA, Pelc MJ, Wang JL, Bowen SJ, Schweitzer BA, Wilcox MV, McDonald J, Smith SE, Foltin S, Rumsey J, Yang Y, Walker MC, Kamtekar S, Beidler D, Thorarensen A. Discovery of novel spirocyclic inhibitors of fatty acid amide hydrolase (FAAH). Part 2. Discovery of 7-azaspiro[3.5]nonane urea PF-04862853, an orally efficacious inhibitor of fatty acid amide hydrolase (FAAH) for pain. Bioorganic and Medicinal Chemistry Letters 2011; 21 (21): 6545-6553.

8. Basappa MP, Sadashiva K, Mantelingu S, Nanjunda S, Rangappa KS. Solution-Phase Synthesis of novel D2isoxazoline libraries via 1,3-dipolar cycloaddition and their antifungal properties. Bioorganic \& Medicinal Chemistry 2003; 11, 4539-4544.

9. Lee Y, Park SM, Kim BH, Synthesis of 5-isoxazol-5yl-20-deoxyuridines exhibiting antiviral activity against HSV and several RNA viruses. Bioorganic \& Medicinal Chemistry Letters 2009; 19, 1126-1128.

10. Talley J, Brown DL, Carter JS, Graneto MJ, Koboldt CM, Masferrer JL, Perkins WE, Rogers RS, Shaffer AF, Zhang Y, Zweifel BS, Seibert K. 4-[5-Methyl-3phenylisoxazol-4-yl]-benzenesulfonamide, Valdecoxib: A potent and selective inhibitor of COX-2. Journal of Medical Chemistry 2000; 43 (5): 775-777.

11. National committee for clinical laboratory standards (NCCLS), 2003, Reference methods for disk difusion antifungal sucseptibility testing of yeasts aproved standard M44-P. Nacional committee for clinical laboratory standards. Wayne, PA.

12. Diba K, Kordbacheh P, Mirhendi Sh, Reazaie S, Mahmouudi M. Identification of Aspergillus species using morphological characteristics. Pakistan Journal of Medical Science 2007; 23, 867-872. 
13. Frederique T, Carlin-Sinclair A, Rene F, Cherton B, Pierre L. Activity and metabolism of cyano-oxime derivatives in various strains of Botrytis cinerea. Pesticide Biochemistry and Physiology 2004; 78, 151-160.

14. Rothe A, Seibold M, Hoppe Th, · Seifert H, Engert A, Caspar C, ' Karthaus M, Tkenheuer G. F Bethe U. Tintelnot $\mathrm{K}$, Cornely OA. Combination therapy of disseminated Fusarium oxysporum infection with terbinafine and amphotericin B. Annals of Hematology 2004; 83, 394-397.

15. Abele E, Abele R, Rubina K, Lukevics E. Novel stereoselective synthesis of E-aryl aldoxime and ketoxime O-ethers. Organic preparations and procedures international 2000; 32 (2): 153-159.

16. Biswanath D, Gurram M, Harish H, Joydeep B. An easy access to 3a,4-dihydro-3H-chromeno[4,3-c]isoxazoles and functionalized isoxazolines. ARKIVOC 2005; 3, 27-35.

17. Kaur J, Singh B, Singal K. Huisgen reaction of nitrile oxides and nitrile imines leading to isoxazoline and pyrazole-4,6-diones. Chemistry of Heterocyclic Compounds 2006; 42 (6): 818-822.

18. Brindaban R, Rajendra ND. Enhanced rate of intramolecular nitrile oxide cycloaddition and rapid synthesis of isoxazoles and isoxazolines. Chemical Monthly 2010; 141, 763-771.

19. Emami S, Falahati M, Banifatemi A, Amanlou M, Shafiee, A. (E)- and (Z)-1,2,4-Triazolylchromanone oxime ethers as conformationally constrained antifungals. Bioorganic \& Medicinal Chemistry 2004; 12, 3971-3976.

20. Ramalingan C, Park Y, Kabilan S. Synthesis, stereochemistry, and antimicrobial evaluation of substituted piperidin-4-one oxime ethers. European Journal of Medicinal Chemistry 2006; 41, 683-696.

21. Ghannoum MA, Rice LB, Antifungal agents: mode of action, mechanisms of resistance, and correlation of these mechanisms with bacterial resistance. Clinical Microbiology Reviews 1999, 12 (4): 501-517. 